\title{
On the Calibration of Segmented Calorimeters
}

\author{
Richard WIGMANS (TTU) \\ CALOR 06, Chicago, June 4 - 9, 2006
}

Outline:

- What is the problem?

- How to deal with it?

- Examples from practice

- Conclusions 


\section{LESSONS FROM 25 YEARS OF R\&D}

- LESSON 1: Energy resolution is determined by fluctuations, not by average values

- LESSON 2: Digital calorimetry has been tried and abandoned, for good reasons

- LESSON 3: A narrow signal distribution is useless if the mean value is incorrect Correct energy scale is at least as as important as good resolution

- LESSON 4: Longitudinal segmentation means asking for (calibration) trouble

- LESSON 5: GEANT based MC simulations of hadronic shower development are fundamentally flawed $\longrightarrow$ useless as design tool

- LESSON 6: If you want to improve hadronic calorimeter performance

$\rightarrow$ reduceleliminate the (effects of) fluctuations that dominate the performance:

i) Fluctuations in the em shower fraction, $f_{\text {em }}$

ii) Fluctuations in visible energy (nuclear binding energy losses) 


\section{Calibration of calorimeter systems}

- Determine relationship between signal (pC, p.e.) and energy $(\mathrm{GeV})$

- Fundamental problem in sampling calorimeters: Different shower components are sampled differently Shower composition changes as shower develops

$\rightarrow$ Sampling fraction changes with the shower age (also E dependent)

How to intercalibrate the sections of a longitudinally segmented calorimeter? 
The sampling fraction changes as shower develops*

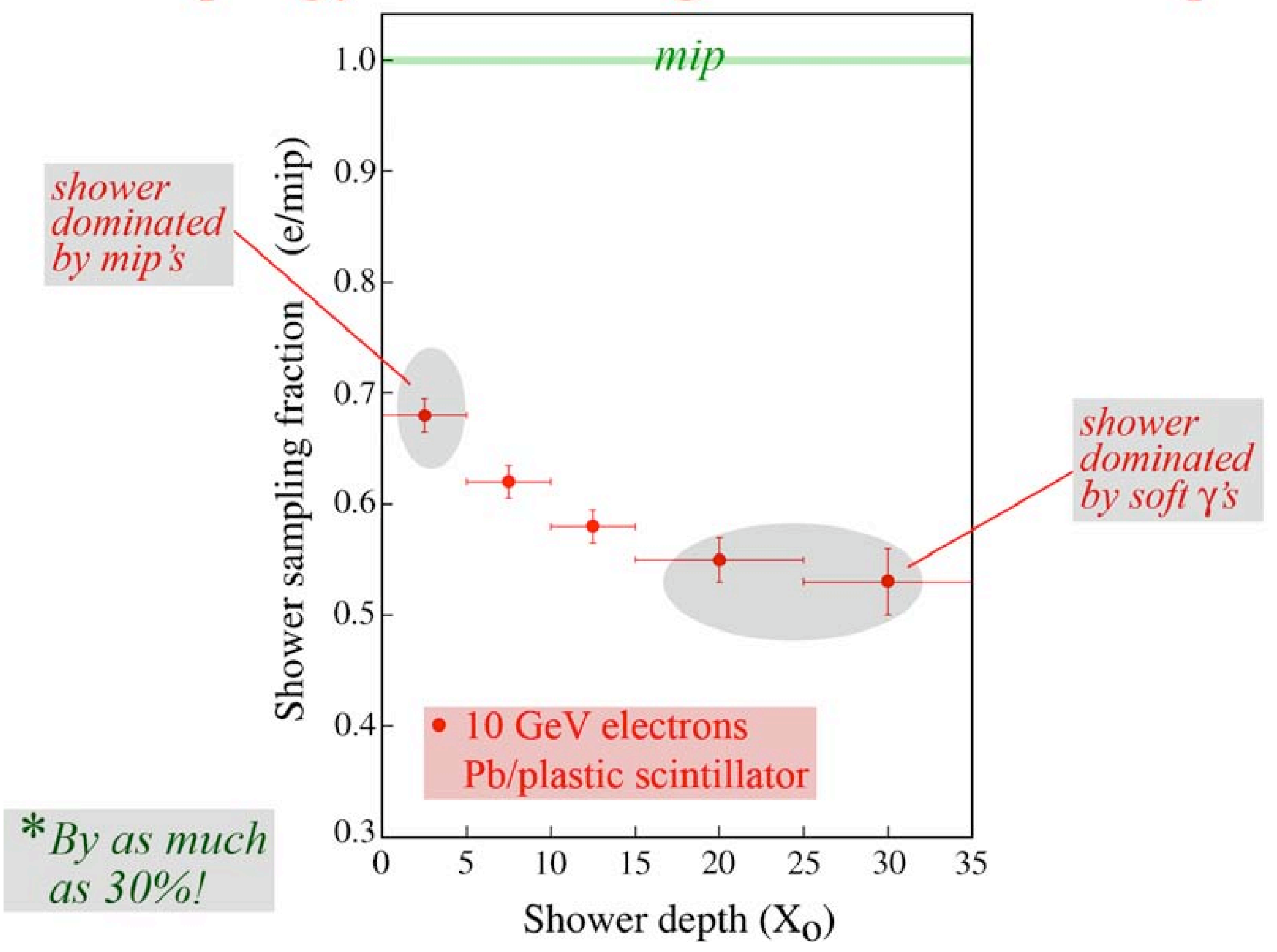




\section{Electromagnetic showers: \\ The importance of SOFT shower particles}

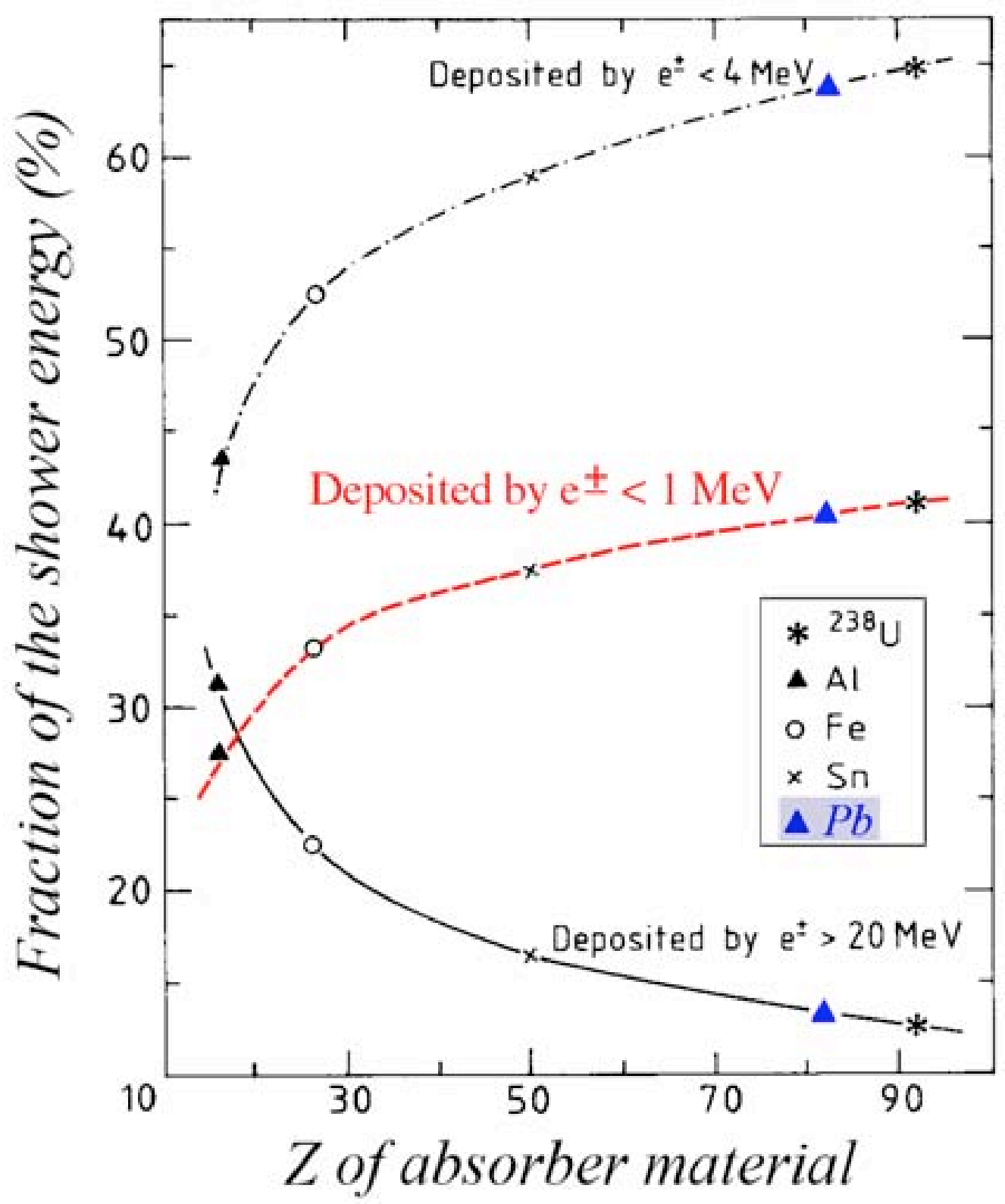

FIG. 2.10.

The composition of em showers.

Shown are the percentages of the energy of $10 \mathrm{GeV}$ electromagnetic showers deposited through shower particles with energies below $1 \mathrm{MeV}$ (dashed curve), below $4 \mathrm{MeV}$ (the dash-dotted curve) or above $20 \mathrm{MeV}$ (the solid curve), as a function of the $Z$ of the absorber material. Results of EGS4 simulations. 
Range of Compton/photoelectrons in calorimeter materials
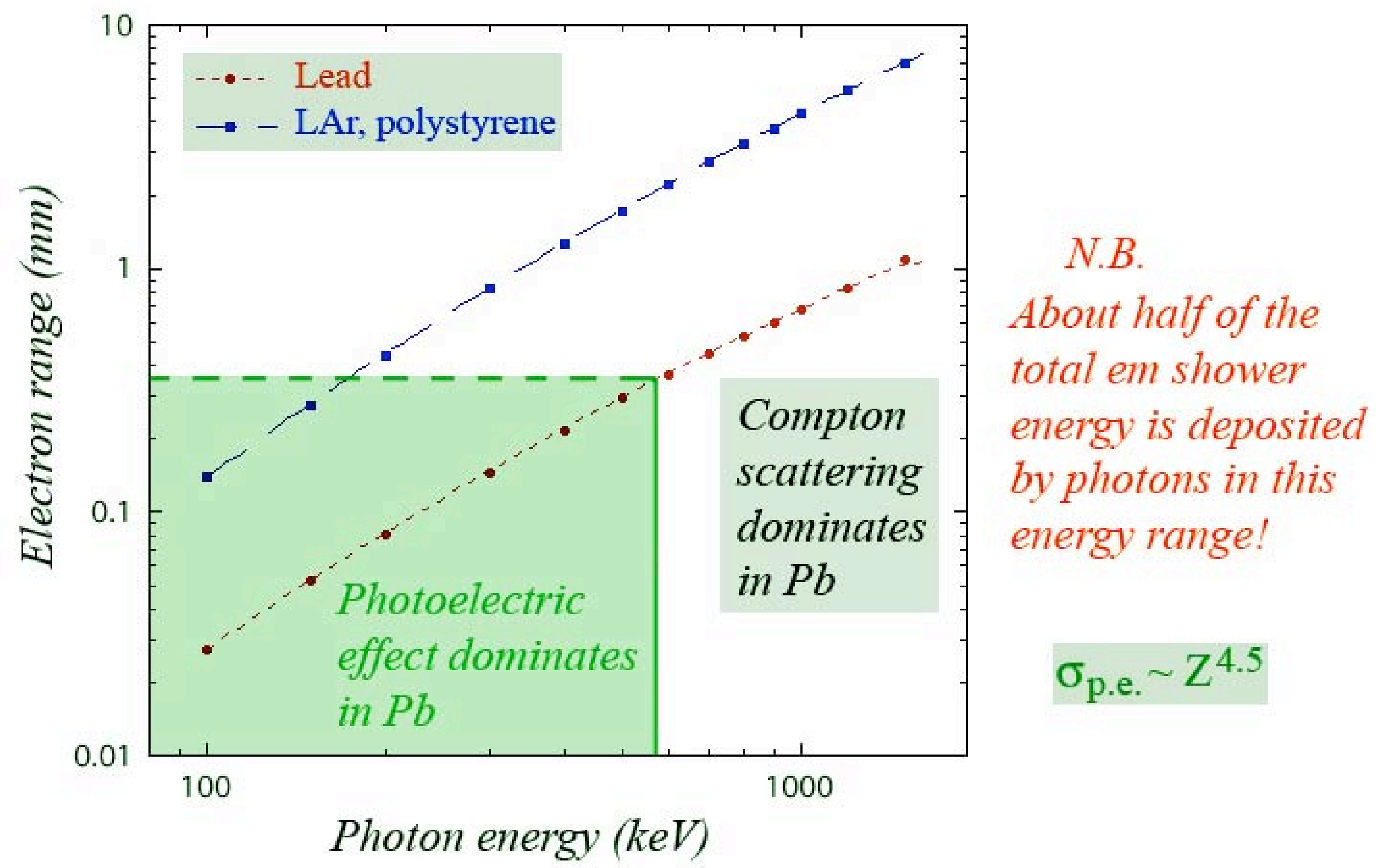


\section{What is the problem? (summary)}

- Shower particles contributing to the signals of sampling calorimeters typically have a range that is much smaller than the distance between sampling layers

$\rightarrow$ Only small fraction of shower particles contribute to signal!

- Signal of a sampling calorimeter is sum of signals from individual contributing shower particles ( $N$ "mips")

- The energy equivalent of one signal unit ("mip") varies with the depth of the shower, in an energy dependent way

- If shower energy is distributed over several calorimeter segments, how to determine then the energy equivalence of the signals in these individual segments??? 


\section{CALIBRATION MISERY}

Consequences of depth dependence sampling fraction

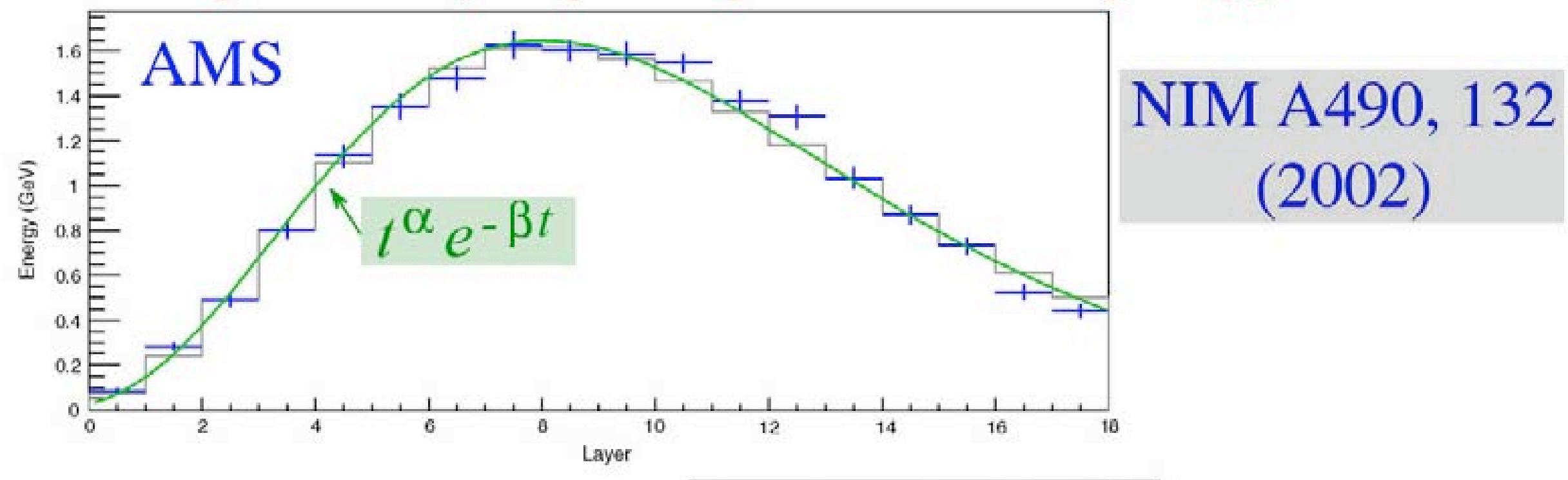

$\mathrm{Pb} /$ scintillating fiber 18 layers $\left(17 \mathrm{X}_{0}\right)$

Calibrated with mip's:

\section{$11.7 \mathrm{MeV} /$ layer}

Shower leakage:

(under)estimated on basis of fit to longitudinal profile

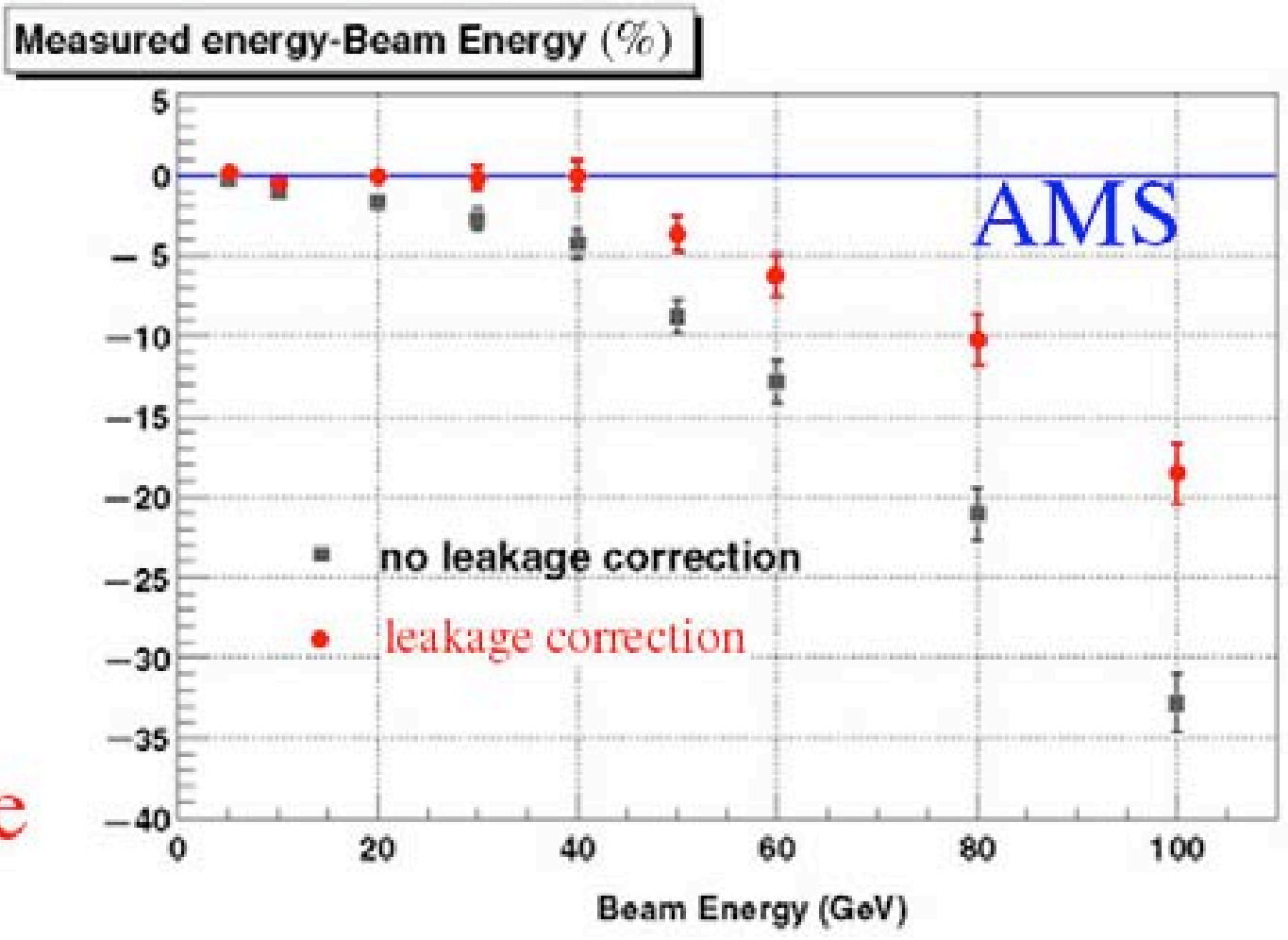


A widely used technique for calibrating segmented devices

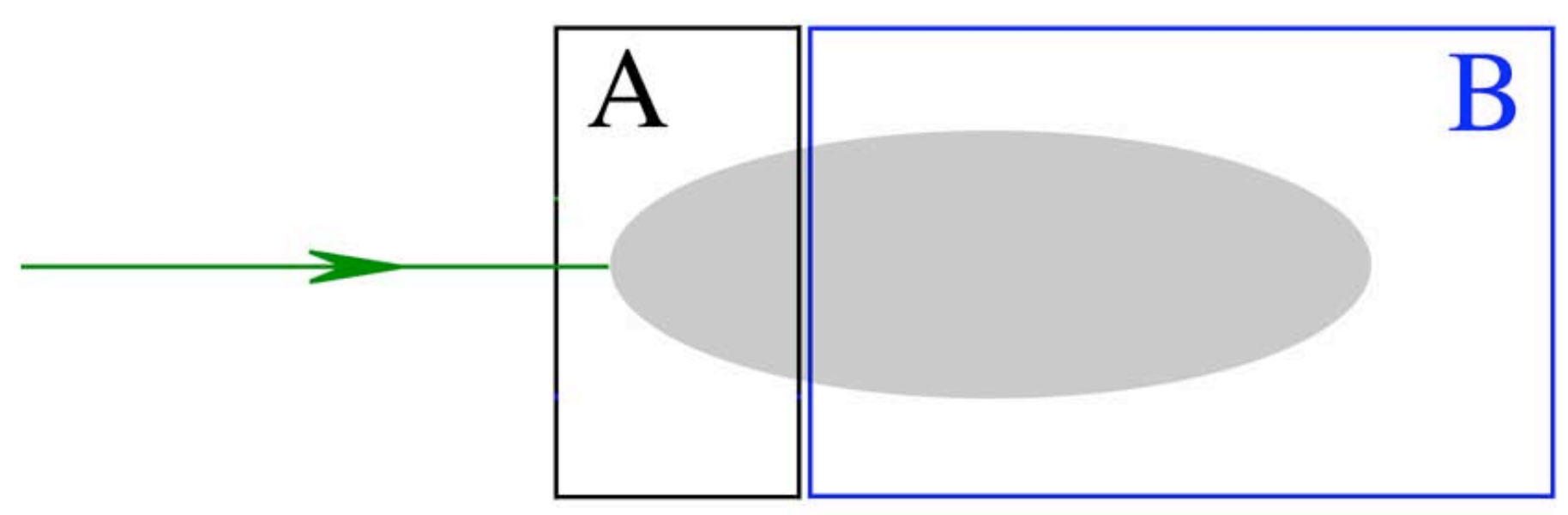

Minimize $\quad Q=\sum_{j=1}^{N}\left[E-A \sum_{i=1}^{n} S_{i j}^{\mathrm{A}}-B \sum_{i=1}^{n} S_{i j}^{\mathrm{B}}\right]^{2}$

$\longrightarrow$ Determine A,B 


\section{Calibrating longitudinally segmented calorimeters}

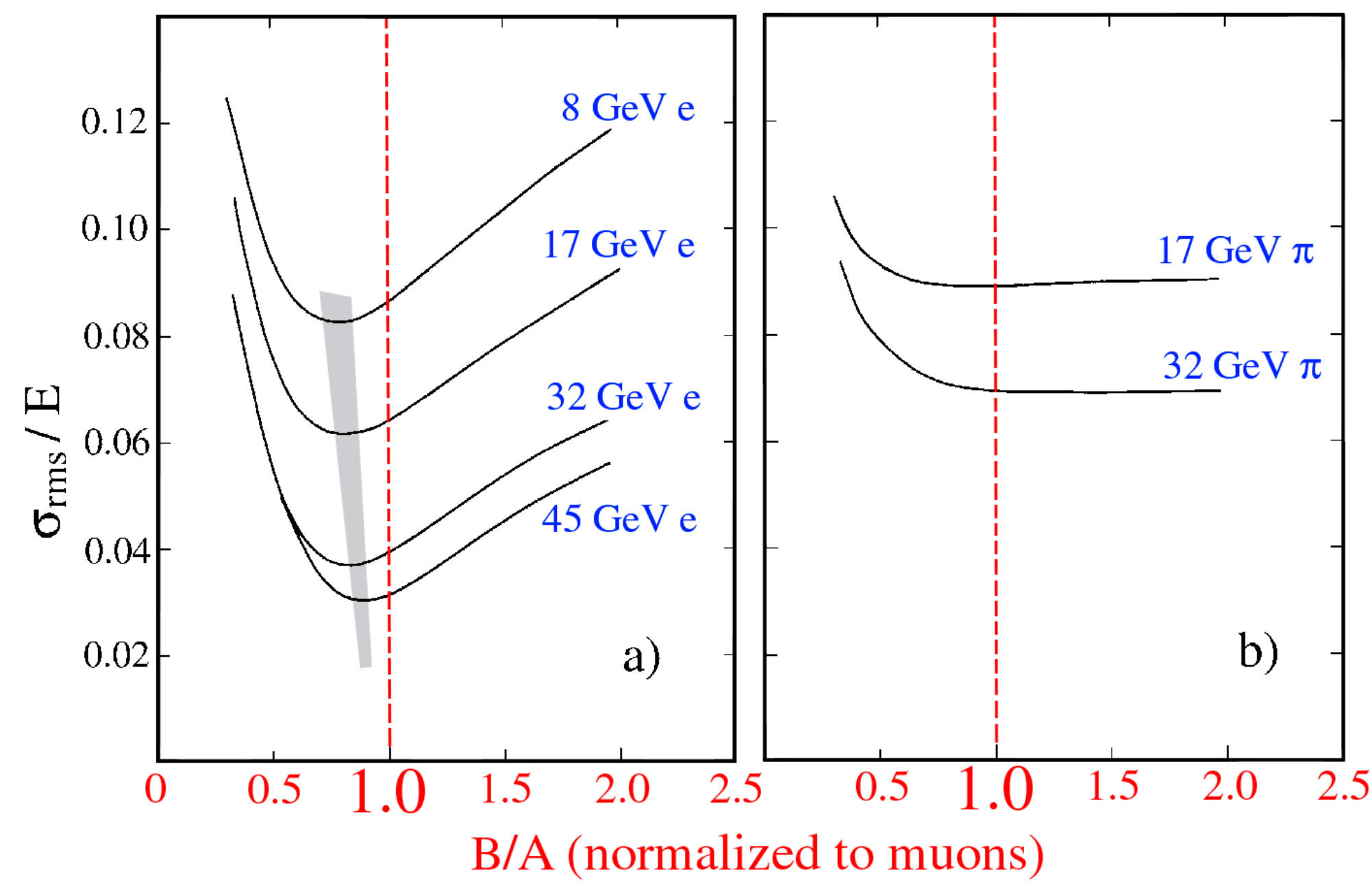

FIG. 6.2. The fractional width $\sigma / E$ of the signal distributions for electrons ( $a$ ) and pions $(b)$ of different energies, as a function of the value of the intercalibration constant $B / A$ of the HELIOS calorimeter system. The dashed line corresponds to the intercalibration constant derived from muon measurements [Ake 87]. 


\section{Results of miscalibration: Non-linearity}

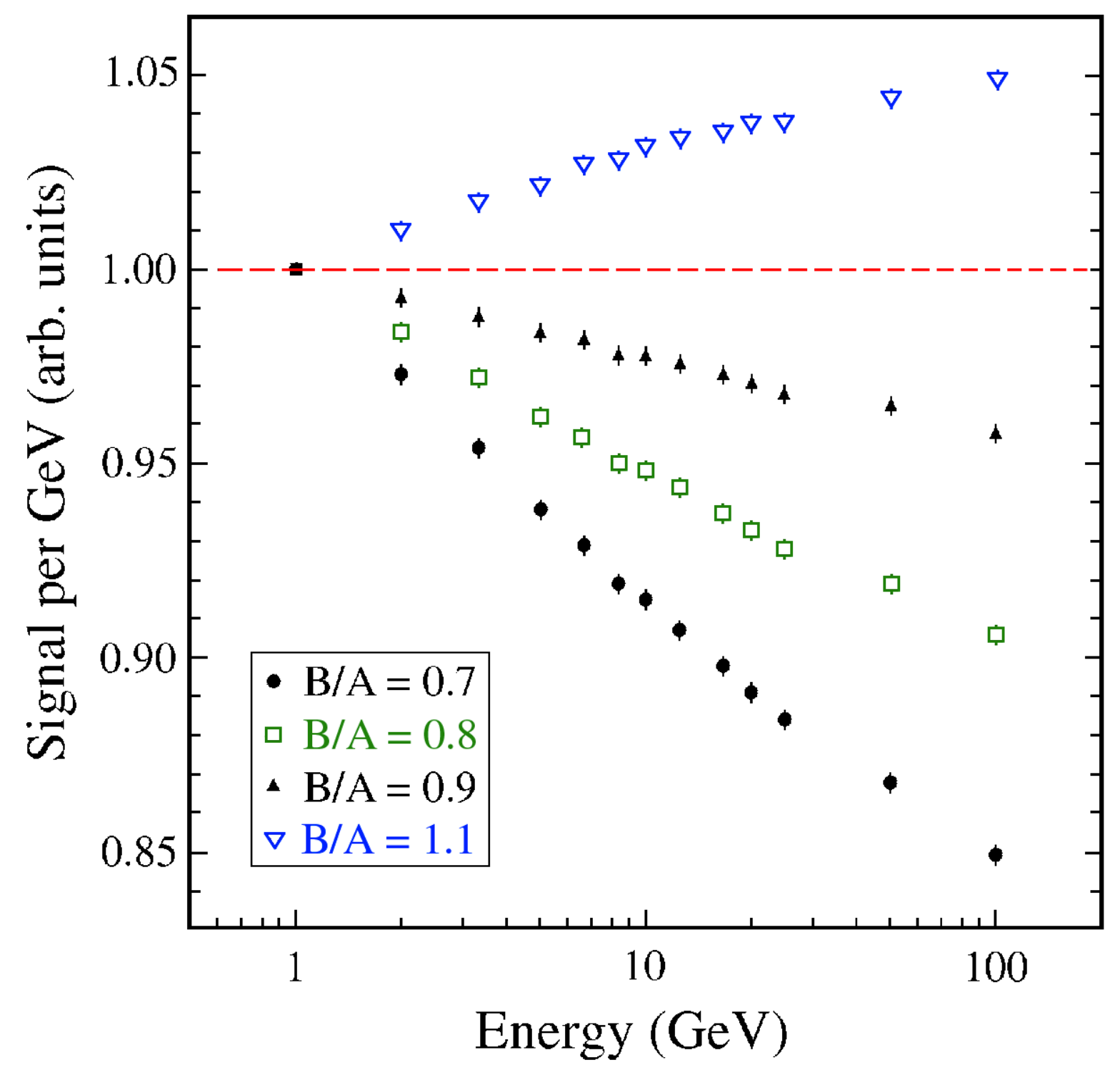

Figure 12: Signal nonlinearity for electrons resulting from miscalibration of a longitudinally segmented calorimeter. The total calorimeter response (average signal per unit of energy) is given for 3 different values of the ratio of the calibration constants for the 2 longitudinal segments, $B / A$. See text for details. 


\section{Results of miscalibration: Mass dependence}

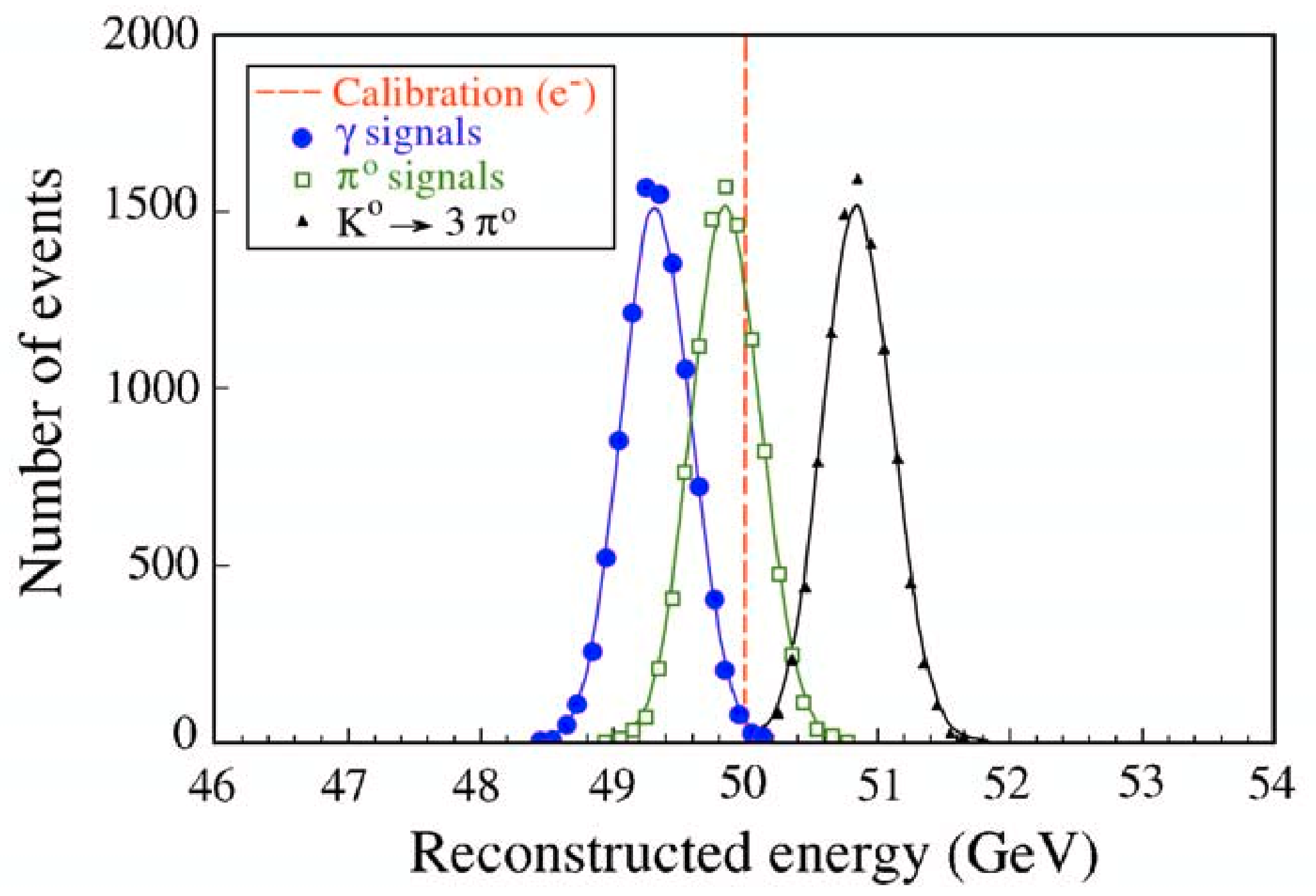

Figure 14: Signal distributions for $\gamma_{\mathbf{S}}$ and various hadrons decaying into all- $\gamma$ final states. All particles have the same nominal energy and the detector, which has an intrinsic resolution of $0.5 \%$ for em showers of this energy, was calibrated with electrons using $B / A=0.8$. See text for details. 


\section{Calibration by Minimizing Total Width}

- Effects of such calibration methods:

- Calibration constants are energy dependent

- Response non-linearity is introduced

- Systematic mismeasurement of energy (e.g., $\pi^{0}, e$ and $\gamma$ of same energy give different measurement results) 


\section{Problems with this method are NOT limited to}

longitudinal segmentation and electromagnetic showers
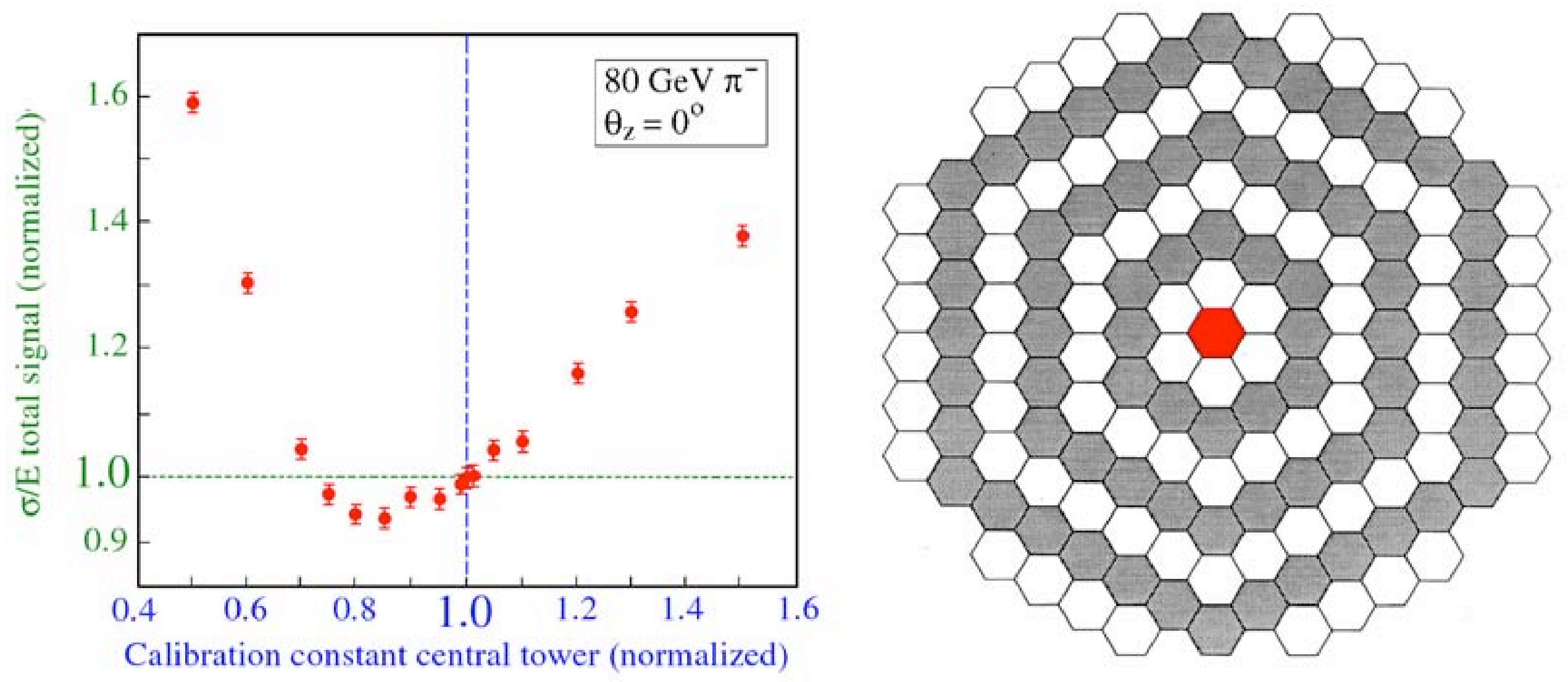

Figure 11: The fractional width, $\sigma / E$, of the signal distribution for $80 \mathrm{GeV} \pi^{-}$in the SPACAL detector as a function of the weighting factor applied to signals from the central calorimeter tower into which the pion beam was steered. The calorimeter towers were calibrated with high-energy electrons [7].

From: NIM A485 (2002) 385. 


\section{Hadronic showers}

- Large fraction of energy is deposited through em showers $\left(\pi^{0}\right)$

- Starting point of the em component(s) fluctuates wildly 


\section{The electromagnetic fraction of hadron showers}

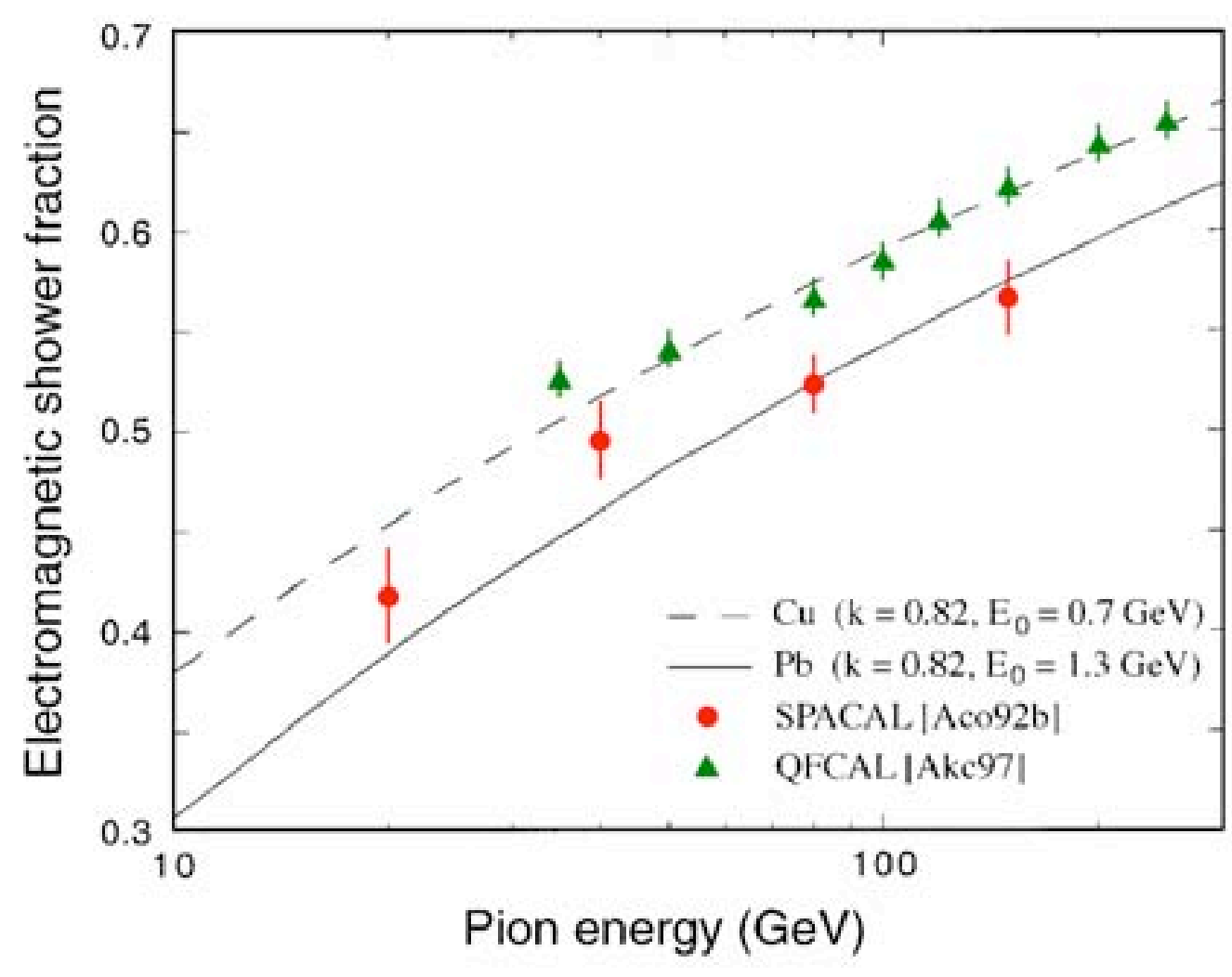

The em fraction is, on average, large and energy dependent

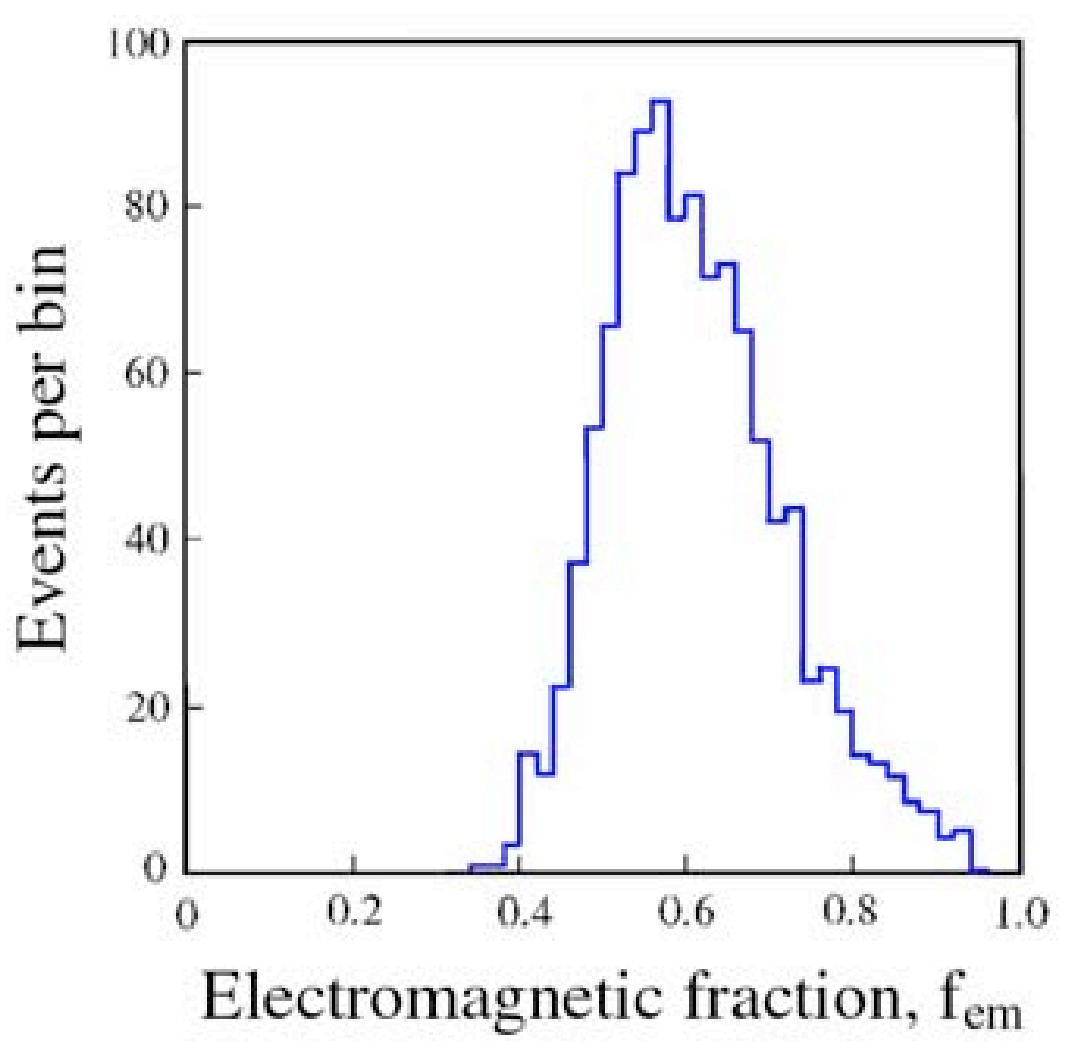

Fluctuations in $\mathrm{fem}_{\mathrm{em}}$ are large and non-Poissonian 


\section{$\pi^{0}$ production is NOT limited to the em section!}
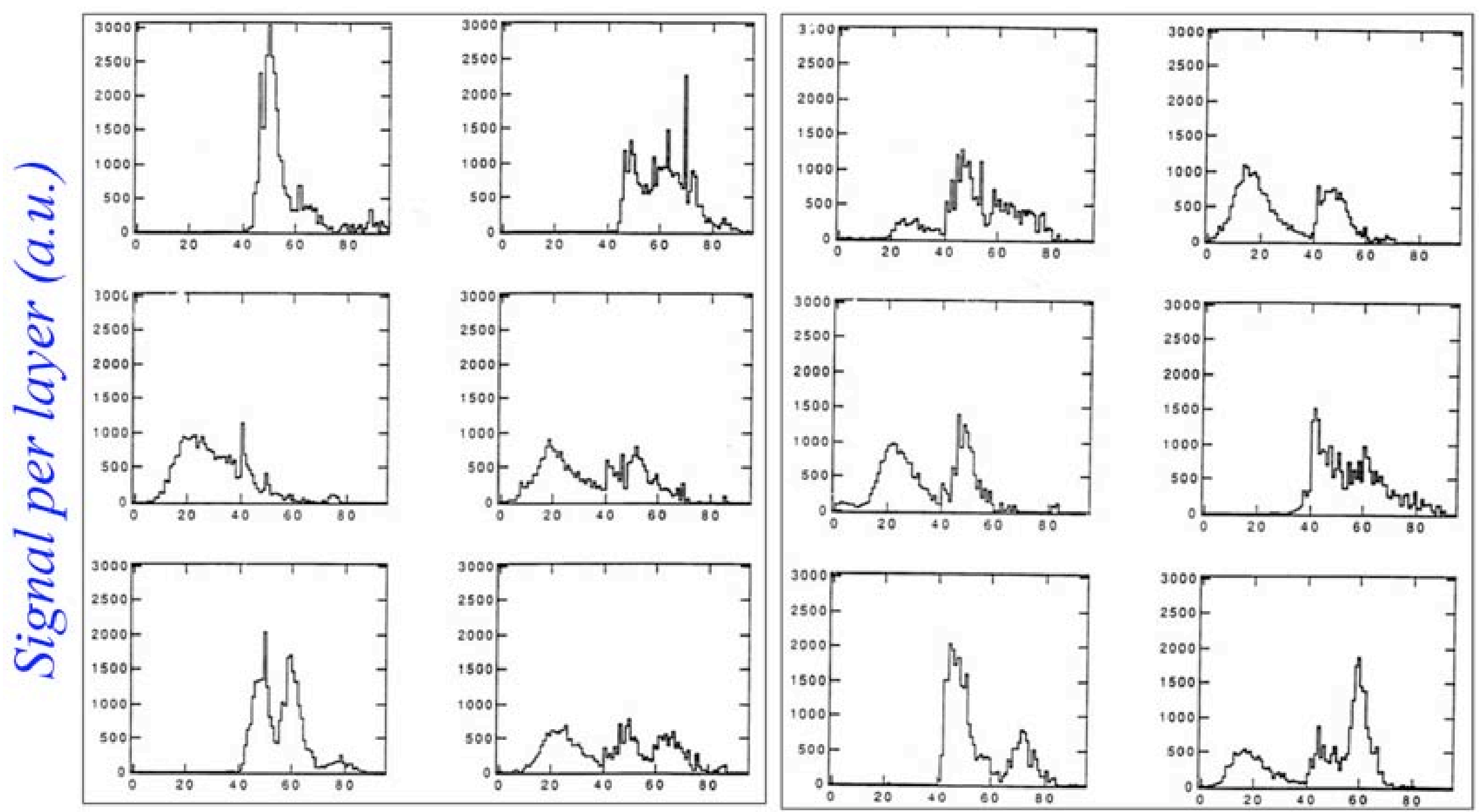

$\operatorname{depth}(0-6 \lambda)$

$270 \mathrm{GeV} \pi$ in $\mathrm{Pb} /$ scintillator (hanging-file experiment) 


\section{Hadronic showers}

- Large fraction of energy is deposited through em showers $\left(\pi^{0}\right)$

- Starting point of the em component(s) fluctuates wildly

- Non-em shower energy primarily deposited by

- spallation protons

- evaporation neutrons

These particles are also sampled very differently than mip's

- In addition, the calorimeter response to the em/non-em components is not the same (e/h $\neq 1$, non-compensation)

$\Rightarrow$ Calibration problems even worse than for em calorimeters 


\section{Alternative method: Each section its own particles}

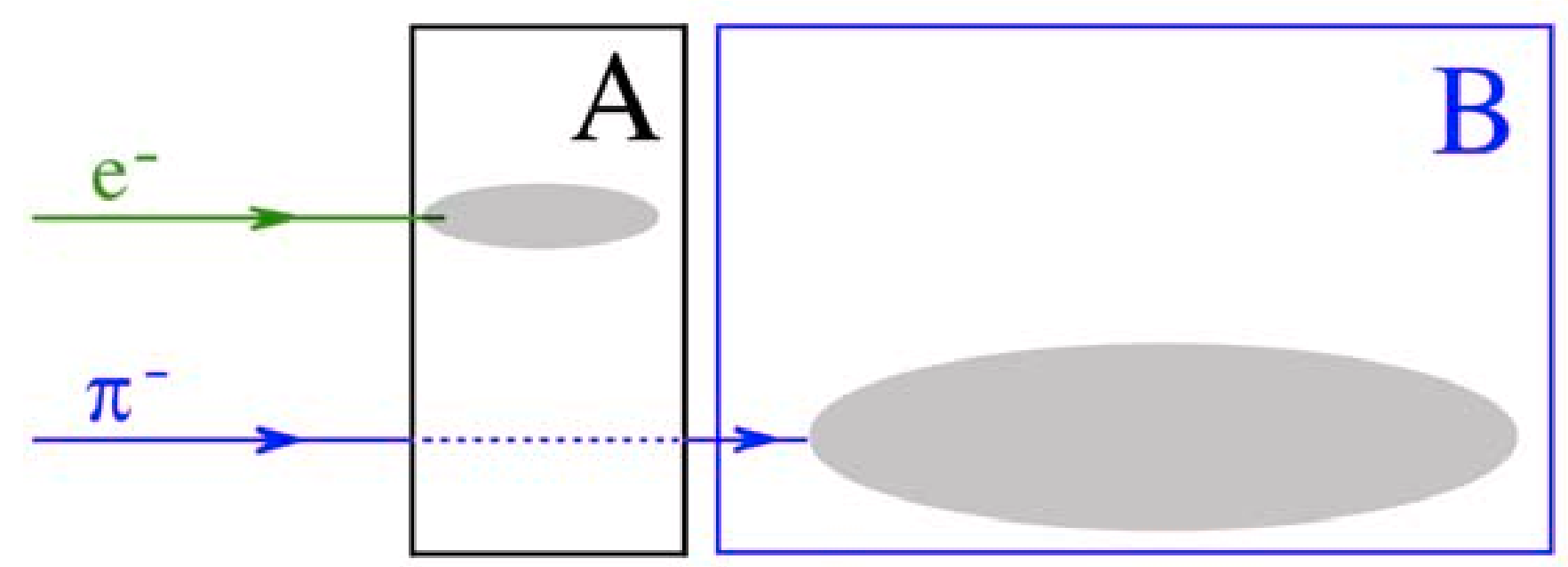

- Problem: How about hadrons that start shower in section A?

- Energy systematically mismeasured depending on $e / h$ values of sections A,B

- Reconstructed energy depends on starting point of shower 


\section{Wrong B/A: Response depends on starting point}
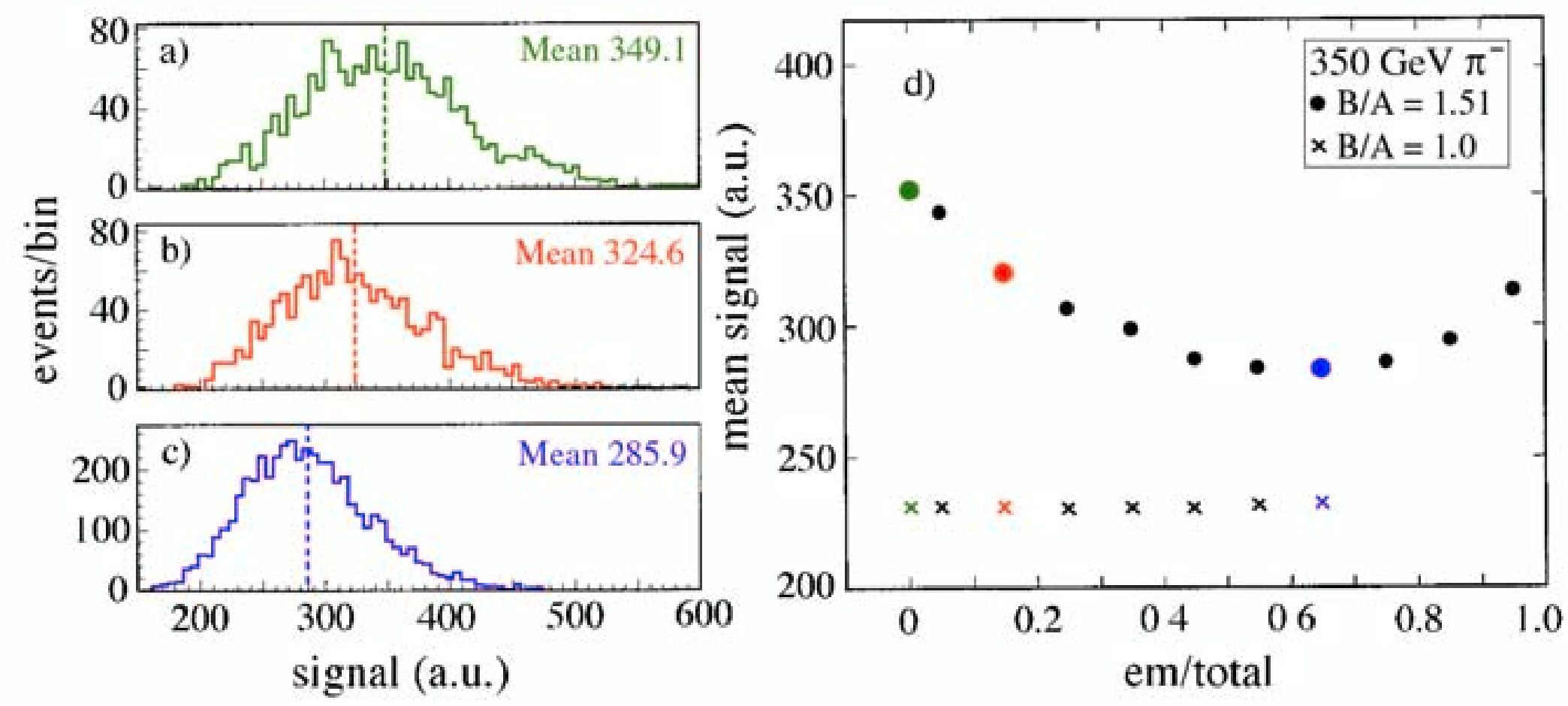

FIG. 6.10. Signal distributions for $350 \mathrm{GeV}$ pion showers in a longitudinally segmented quartz-fiber calorimeter, for events in which different fractions of the (unweighted) shower energy were recorded in the em calorimeter section. Shown are distributions for which this fraction was compatible to zero $(a), 10-20 \%(b)$, or $60-80 \%(c)$. The average calorimeter signal for $350 \mathrm{GeV}$ pions, as a function of this fraction, is shown in diagram $(d)$. The calorimeter was calibrated on the basis of $B / A=1.51$ in all these cases, as required for reconstructing the energy of $350 \mathrm{GeV}$ pions that penetrated the em compartment without undergoing a strong interaction. Diagram $(d)$ also contains results (the crosses) obtained for a calorimeter calibration on the basis of $B / A=1$. From [Gan 98]. 


\section{Preliminary conclusions}

- Calibration is a very delicate issue

- Discussed strategies (and several others used in practice) only work for a subset of events

(electrons of certain energy, pions penetrating em section, ...)

- Negative consequences for the rest of the events

- Systematic mismeasurement of energy

- Reconstructed energy depends on starting point shower

- Signal non-linearity, .....

- A more correct method: $\mathrm{B} / \mathrm{A}=1$ i.e. calibrate all calorimeter sections in the same way

$$
\text { However....... }
$$




\section{CALIBRATION MISERY}

Consequences of depth dependence sampling fraction

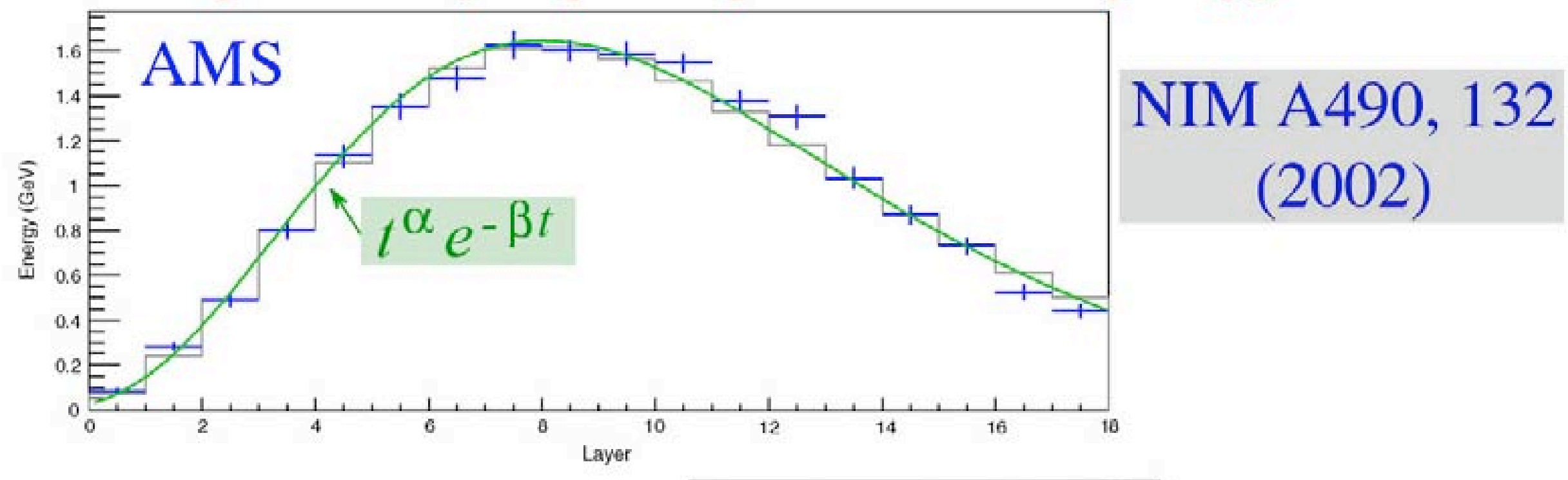

$\mathrm{Pb} /$ scintillating fiber 18 layers $\left(17 \mathrm{X}_{0}\right)$

Calibrated with mip's:

\section{$11.7 \mathrm{MeV} /$ layer}

Shower leakage:

(under)estimated on basis of fit to longitudinal profile

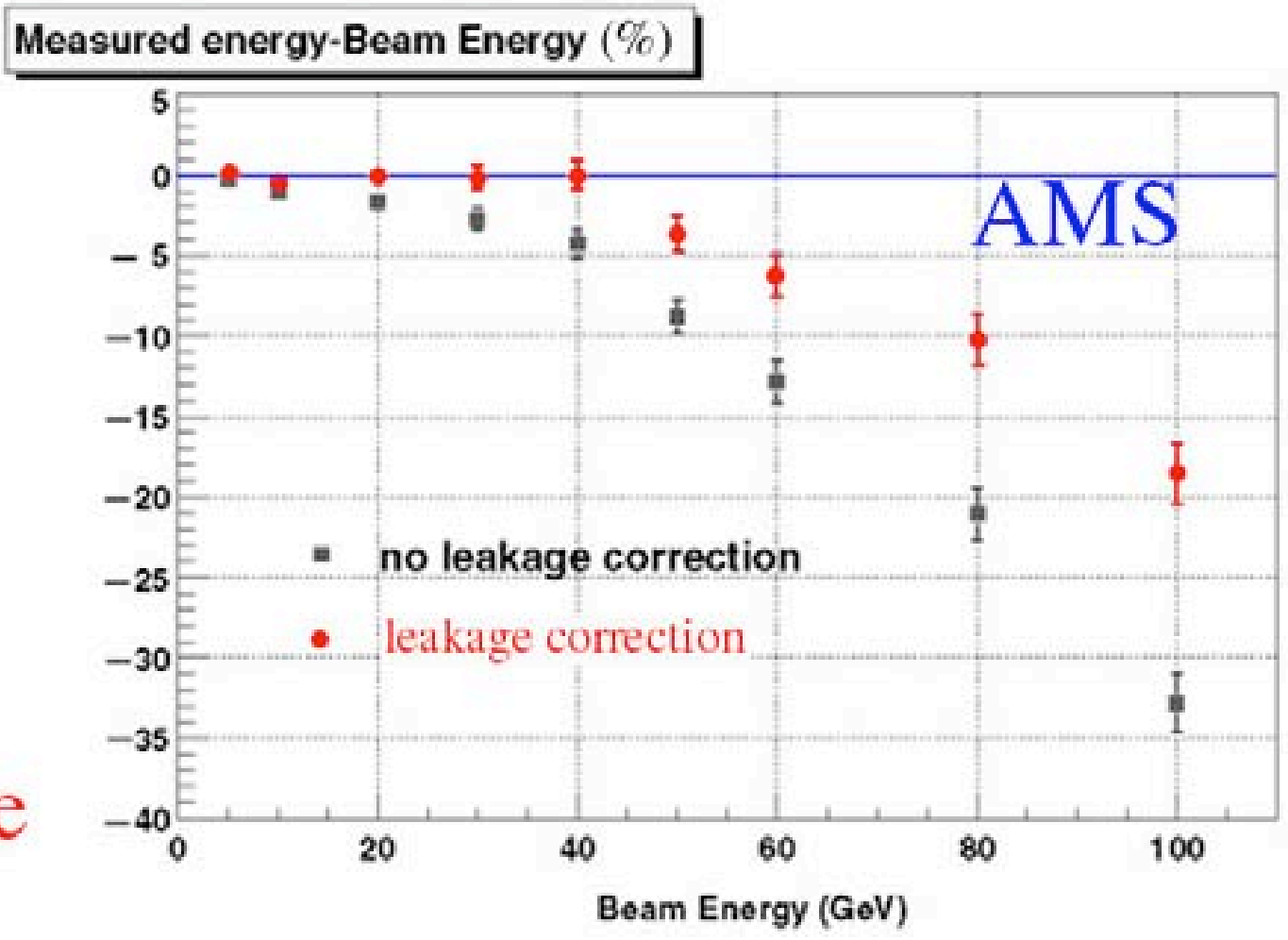




\section{So what to do?}

- Determine the calibration constants of the longitudinal segments on the basis of

Monte Carlo simulations!!! 


\section{ATLAS: The longitudinally segmented (LAr) ECAL}

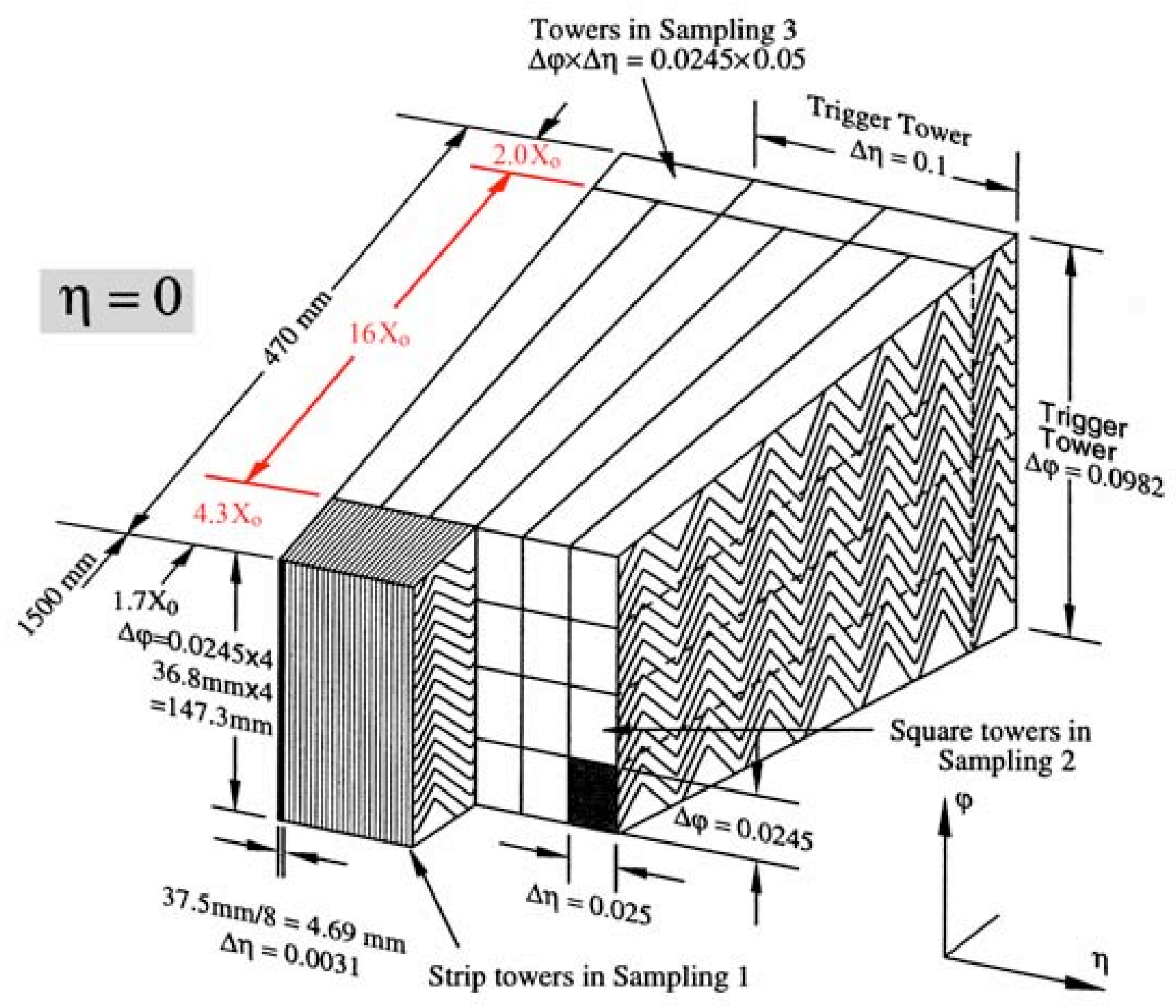




\section{ATLAS: Depth dependent em sampling fraction}
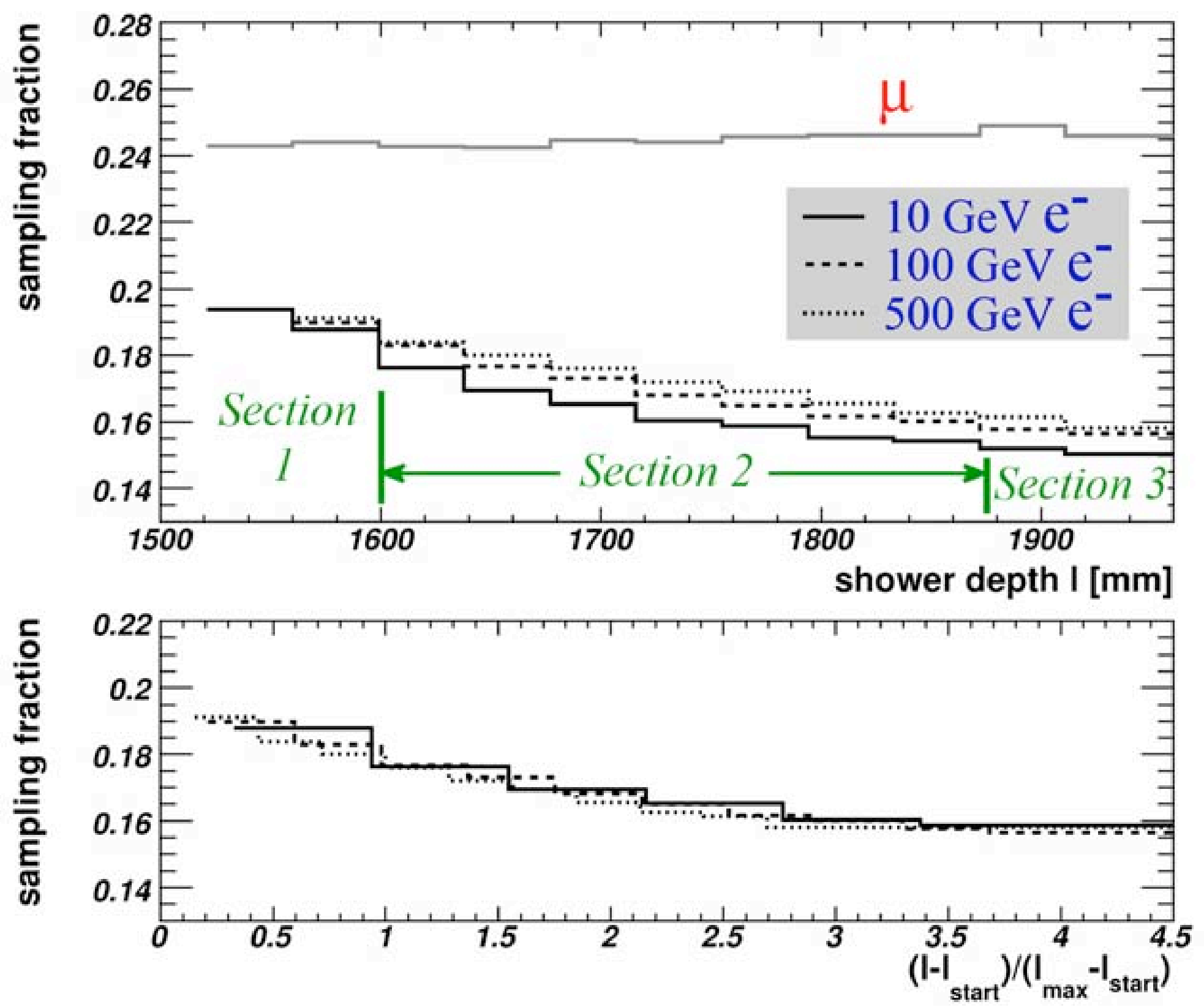


\section{ATLAS: Energy reconstruction ECAL}
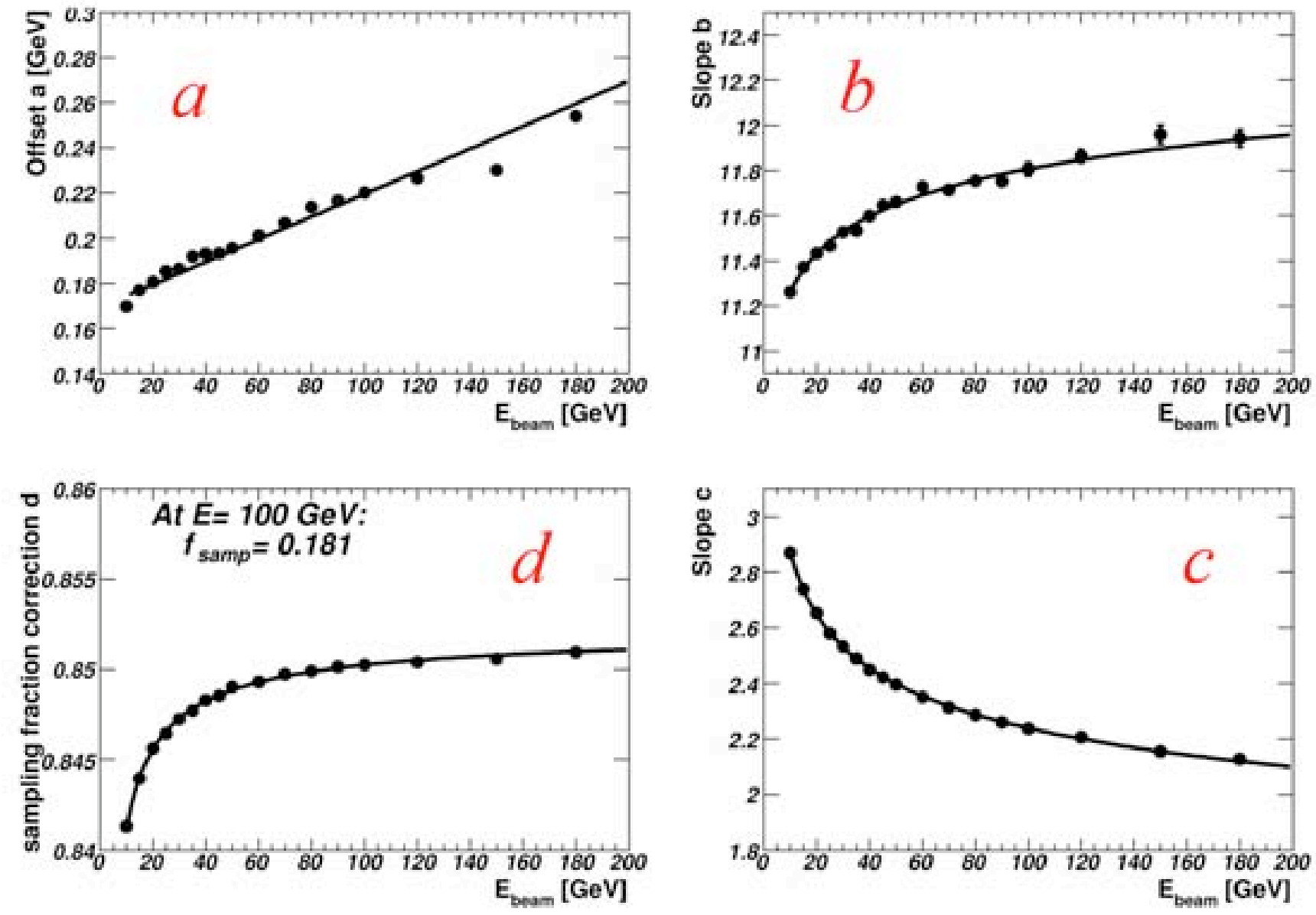

$E^{r e c}=\left(a(E)+b(E) E_{0}^{v i s}+c(E)\left(E_{0}^{\text {vis }} \cdot E_{1}^{\text {vis }}\right)^{0.5}+\frac{1}{d(E) f_{\text {samp }}} \sum_{i=1,3} E_{i}^{\text {vis }}\right) \cdot f_{\text {cell impact }}(\Delta \Phi) \cdot\left(1+f_{\text {leakage }}\right)$ 


\section{ATLAS: Electromagnetic signal linearity}

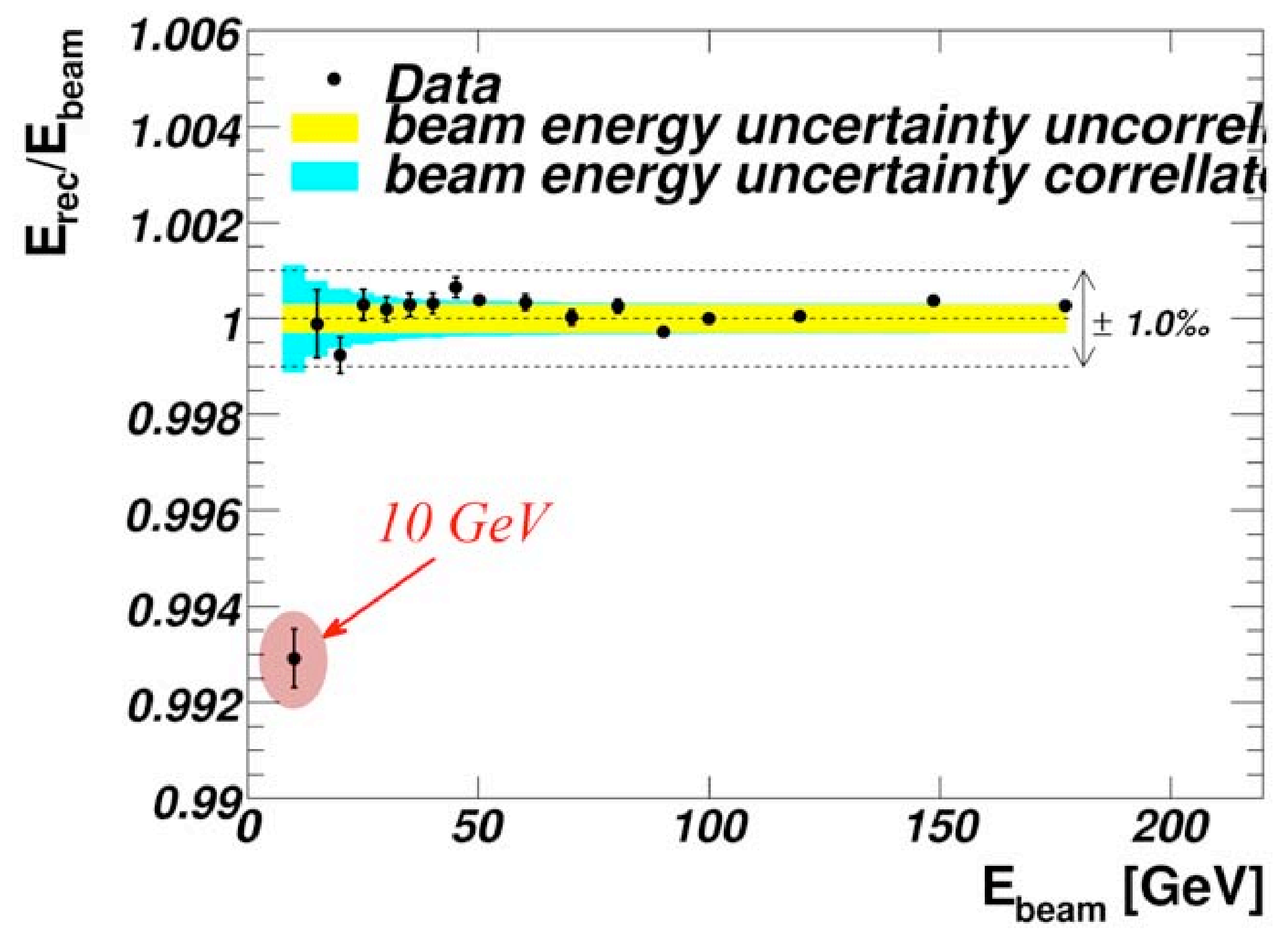




\section{However, this scheme is only valid for electrons!}

\section{(elctron showers $\neq$ photon showers $\neq \pi^{0}$ showers)}

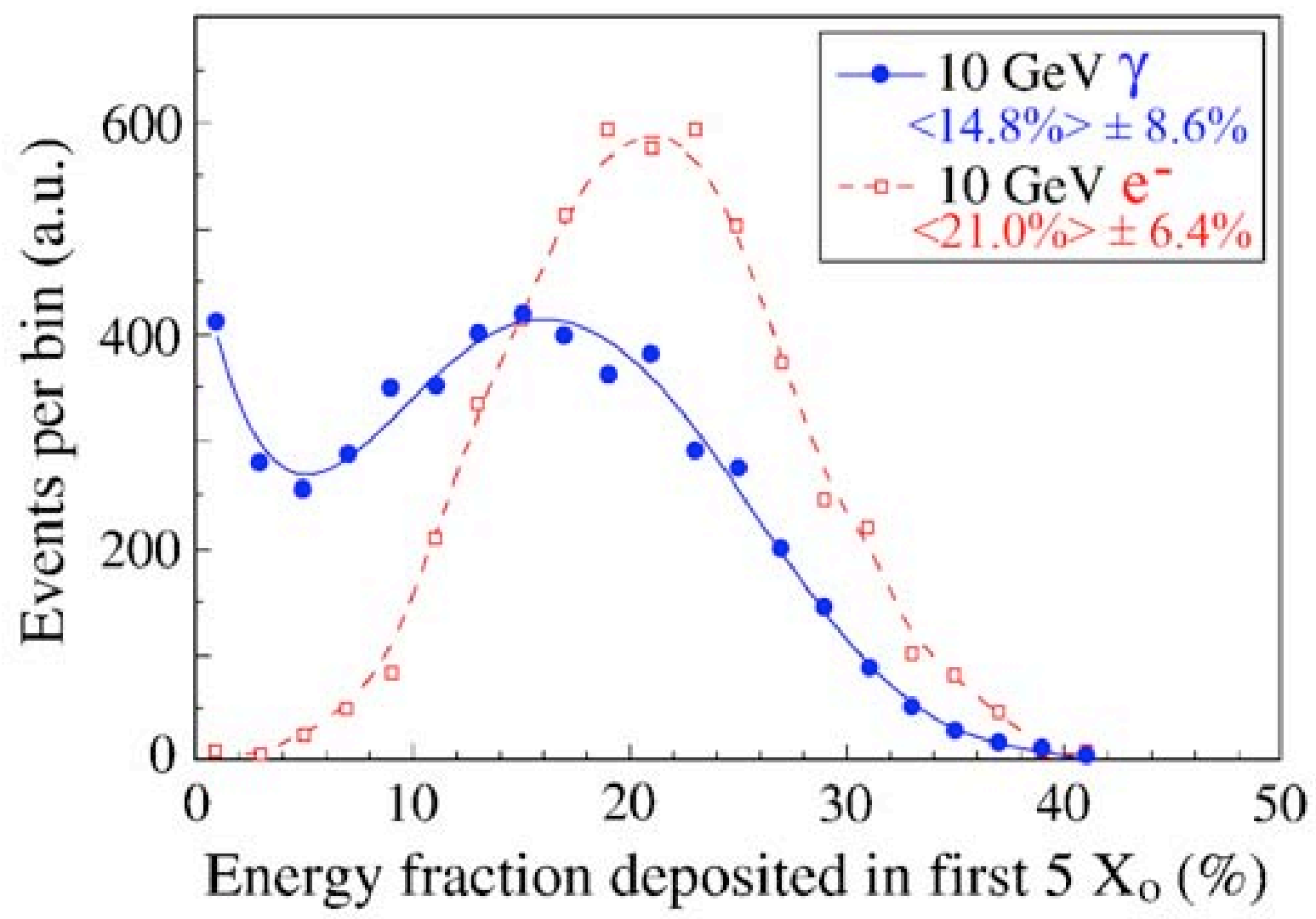

Distribution of the energy fraction deposited in the first 5 radiation lengths by $10 \mathrm{GeV}$ electrons and $\gamma \mathrm{s}$ showering in lead. Results of EGS4 simulations.

From: NIM A485 (2002) 385 


\section{Some final thoughts}

- Calibration of a longitudinally segmented sampling calorimeter is a nightmare

- I don't know how to do it

- More sections $\longrightarrow$ more problems

\section{How to avoid these problems??}

- No longitudinal segmentation (what's the purpose anyway?)

- Use homogeneous calorimeters

- Use sampling calorimeters with $Z_{\mathrm{abs}} \approx Z_{\mathrm{act}}$ 